\title{
Geometry of Multiflavor Galileon-Like Theories
}

\author{
Mark P. Bogers* and Tomáš Brauner \\ Department of Mathematics and Physics, University of Stavanger, 4036 Stavanger, Norway
}

(Received 22 February 2018; revised manuscript received 29 June 2018; published 25 October 2018)

\begin{abstract}
We use Lie-algebraic arguments to classify Lorentz-invariant theories of massless interacting scalars that feature coordinate-dependent redundant symmetries of the Galileon type. We show that such theories are determined, up to a set of low-energy effective couplings, by specifying an affine representation of the Lie algebra of physical, nonredundant internal symmetries and an invariant metric on its target space. This creates an infinite catalog of theories relevant for both cosmology and high-energy physics thanks to their special properties such as enhanced scaling of scattering amplitudes in the soft limit.
\end{abstract}

DOI: 10.1103/PhysRevLett.121.171602

Introduction.-Theories of the Galileon type have aroused immense attention in the context of modified gravity [1]. The simplest example of a Galileon field theory contains a massless scalar field $\theta$ and a single interaction term, $\left(\partial_{\mu} \theta\right)^{2} \square \theta$. It has a number of remarkable properties. First, its Lagrangian density is invariant up to a surface term under the coordinate-dependent "Galilean" shift, $\theta(x) \rightarrow \theta(x)+\alpha+\beta_{\mu} x^{\mu}$, where $\alpha$ and $\beta_{\mu}$ are constant parameters. Second, the corresponding equation of motion is of second order, implying absence of instabilities due to Ostrogradski ghosts [2]. An attractive feature for cosmological model building is that the Galilean symmetry together with the requirement of second-order equation of motion strongly restricts the action, leaving only five possible Lagrangians in four spacetime dimensions [3] (see, however, Refs. [4,5] for a recent discussion of observational constraints on Galileon-like theories).

Besides cosmology, the single-field Galileon theory is also interesting for high-energy physics. Indeed, all the five possible Lagrangians can be interpreted as so-called WessZumino (WZ) terms [6] of the Galilean shift symmetry [7]. Consequently, they are free from radiative corrections and remain exact on the quantum level [8,9]. Moreover, the five Galileon Lagrangians turn out to be related by a set of duality transformations [10]. Their special linear combination features a nontrivial hidden symmetry [11]. Finally, scattering amplitudes of the Galileon field $\theta$ vanish in the limit where the momentum of one of the participating particles goes to zero (the "soft limit"). This alone follows from the fact that $\theta$ can be interpreted as a NambuGoldstone (NG) boson of the shift symmetry $\theta \rightarrow \theta+\alpha$

Published by the American Physical Society under the terms of the Creative Commons Attribution 4.0 International license. Further distribution of this work must maintain attribution to the author(s) and the published article's title, journal citation, and DOI. Funded by SCOAP.
[12]. However, the presence of the Galilean shift symmetry makes the soft limit enhanced: the scattering amplitudes vanish with the second power of momentum. The hidden symmetry of the special Galileon further enhances its scattering amplitudes so that they scale with the third power of momentum [13]. These features grant the Galileon a central position in the rapidly developing branch of high-energy physics, which studies scattering amplitudes of massless particles [14]. Theories whose scattering amplitudes feature an enhanced soft limit are so strongly constrained that their complete classification seems feasible $[13,15,16]$.

The outstanding features of the original Galileon theory [3] prompted a search for its generalizations, inspired by both its potential for cosmology and the desire to understand to what extent it is uniquely determined by its properties. It has been shown that the requirement of second-order equation of motion admits a much broader class of solutions than the five WZ terms with Galilean symmetry [17]. Some modifications of the Galileon theory, preserving its special properties in a curved spacetime, were developed in Ref. [18].

A vast new horizon opens by considering Galileonlike theories with multiple massless fields. The search for such theories, motivated by applications to cosmology, was initiated in Ref. [19] and continued, for instance, in Ref. [20], following the equation of motion line of thought. A field-theoretic approach based on symmetry considerations was taken in Refs. [9,21], leading to some straightforward multiflavor generalizations of the original Galileon theory. The authors of Refs. [13,16] put forward a systematic bottom-up approach from the scattering amplitude point of view. However, their technique, based on a recursive analysis of tree-level scattering amplitudes, makes it difficult to draw general conclusions, valid for an arbitrary number of field species.

Our aim in this Letter is to classify multiflavor Galileonlike theories from the symmetry point of view. Note that the 
Galilean shift symmetry $\theta(x) \rightarrow \theta(x)+\alpha+\beta_{\mu} x^{\mu}$ does not commute with the Poincare group and, as such, has to be spontaneously broken [22]. Yet, it does not give rise to an independent NG mode. Such symmetries are dubbed "redundant" and have been studied intensively [23]. Taking the presence of a redundant symmetry as the defining property of a "Galileon-like" theory leads to the following question: given a set of massless scalars-NG bosons-and the associated physical internal symmetry, what additional, redundant symmetries can be consistently imposed on the system? We answer this question by solving the Lie-algebraic consistency constraints on the commutators of the symmetry generators. Furthermore, we construct three concrete classes of theories satisfying all the symmetry constraints, two of which generalize the known multi-Galileon and multiflavor Dirac-Born-Infeld (DBI) theories. As a corollary of our Letter, we map the landscape of theories of multiple massless scalars whose scattering amplitudes feature an enhanced soft limit [24].

Geometry of redundant symmetries. - Consider a Poincaré-invariant theory of one or more massless scalars (NG bosons). Its symmetry generators include the angular momentum $J_{\mu \nu}$ and momentum $P_{\mu}$, along with the internal symmetry generators $Q_{i}$, which are assumed to satisfy $\left[P_{\mu}, Q_{i}\right]=0$. This condition holds for most Lorentz-scalar symmetries in physics; its violation tends to lead to nonvanishing scattering amplitudes in the soft limit [25]. With this restriction, the commutators of all the listed generators are fixed by Lorentz invariance, except for the internal symmetry algebra $\left[Q_{i}, Q_{j}\right]=i f_{i j}^{k} Q_{k}$. How can the symmetry be augmented with a set of additional, redundant generators? It is known [26] that, for the new generators to be redundant, their commutator with $P_{\mu}$ must be a linear combination of $Q_{i}$, so they must be Lorentz vectors [27]. A trivial example is the linear Galilean shift, corresponding to the parameter $\beta_{\mu}$.

We therefore add to our theory a set of generators $K_{\mu A}$, the index $A$ indicating their multiplet structure, related in some yet unknown way to the algebra of $Q_{i}$. Lorentz invariance now fixes the value of $\left[J_{\mu \nu}, K_{\lambda A}\right]$ and restricts the remaining commutators among the generators to

$$
\begin{aligned}
{\left[P_{\mu}, K_{\nu A}\right] } & =i\left(a_{A}^{i} g_{\mu \nu} Q_{i}+b_{A} J_{\mu \nu}+\bar{b}_{A} \epsilon_{\mu \nu \kappa \lambda} J^{\kappa \lambda}\right), \\
{\left[K_{\mu A}, K_{\nu B}\right] } & =i\left(g_{A B} J_{\mu \nu}+\bar{g}_{A B} \epsilon_{\mu \nu \kappa \lambda} J^{\kappa \lambda}+c_{A B}^{i} g_{\mu \nu} Q_{i}\right), \\
{\left[K_{\mu A}, Q_{i}\right] } & =i\left(d_{A i} P_{\mu}+e_{A i}^{B} K_{\mu B}\right),
\end{aligned}
$$

with as yet undetermined coefficients $a_{A}^{i}, \ldots$ In order for the addition of the generators $K_{\mu A}$ to be consistent, the full Lie algebra must satisfy a set of Jacobi identities. A straightforward calculation shows that $b_{A}=\bar{b}_{A}=$ $\bar{g}_{A B}=0$. The remaining coefficients can be nonzero, but are restricted by a set of conditions that can be equivalently expressed as follows (see the Supplemental Material [29]).
First, define a set of linear combinations

$$
Q_{A} \equiv a_{A}^{i} Q_{i}, \quad Q_{A B} \equiv c_{A B}^{i} Q_{i}
$$

The commutation relations between $P_{\mu}, K_{\mu A}, Q_{A}$, and $Q_{A B}$ necessarily take the form

$$
\begin{aligned}
{\left[P_{\mu}, K_{\nu A}\right] } & =i g_{\mu \nu} Q_{A}, \\
{\left[K_{\mu A}, K_{\nu B}\right] } & =i\left(g_{A B} J_{\mu \nu}+g_{\mu \nu} Q_{A B}\right), \\
{\left[K_{\mu A}, Q_{B}\right] } & =-i g_{A B} P_{\mu}, \\
{\left[K_{\mu C}, Q_{A B}\right] } & =i\left(g_{A C} K_{\mu B}-g_{B C} K_{\mu A}\right), \\
{\left[Q_{A}, Q_{B}\right] } & =0, \\
{\left[Q_{A B}, Q_{C}\right]=} & i\left(g_{B C} Q_{A}-g_{A C} Q_{B}\right), \\
{\left[Q_{A B}, Q_{C D}\right]=} & i\left(g_{A D} Q_{B C}+g_{B C} Q_{A D}\right. \\
& \left.-g_{A C} Q_{B D}-g_{B D} Q_{A C}\right) .
\end{aligned}
$$

The first commutator is nothing but a multiflavor generalization of the Galileon algebra. We therefore expect $Q_{A}$ to play the role of shift symmetries, acting on a space of Galileon-like fields $\theta^{A}$, which we will refer to as the "Galileon space." Next, introduce the set of block matrices

$$
\left(T_{i}\right)_{B}^{A} \equiv\left(\begin{array}{c|c}
-i e_{B i}^{A} & 0 \\
\hline d_{B i} & 0
\end{array}\right)
$$

These define a set of affine maps (combinations of linear transformations and translations) on a vector space, isomorphic to the Galileon space. Given that $\left[T_{i}, T_{j}\right]=i f_{i j}^{k} T_{k}$, the matrices $T_{i}$ generate an affine representation of the Lie algebra of $Q_{i}$. In addition, they define the adjoint action of $Q_{i}$ on $Q_{A}$ and $Q_{A B}$. By grouping the latter two as

$$
L_{A B} \equiv\left(\begin{array}{c|c}
Q_{A B} & i Q_{A} \\
\hline-i Q_{B} & 0
\end{array}\right),
$$

we get an object that transforms as a rank-2 antisymmetric tensor under the representation $T_{i}$, that is,

$$
\left[Q_{i}, L_{A B}\right]=\left(T_{i}^{T} L+L T_{i}\right)_{A B} .
$$

Altogether, the most general symmetry algebra, obtained by augmenting an internal symmetry with a set of redundant generators $K_{\mu A}$, is fully determined by (i) the algebra of internal generators $Q_{i}$, (ii) its affine representation $T_{i}$, and (iii) the matrix $g_{A B}$, which forms an invariant rank-2 symmetric tensor of the representation $T_{i}$.

The last four lines in Eq. (3) are a facsimile of the Poincaré algebra, except that they refer to the Galileon space and use the metric $g_{A B}$. Altogether, the commutators listed in Eq. (3) together with those of the Poincare group admit a remarkable geometric interpretation: they generate 
the group of isometries of the direct sum of the flat Minkowski spacetime and the Galileon space, equipped with the metric $g_{\mu \nu} \oplus g_{A B}$. The generators $Q_{A}$ naturally represent translations and $Q_{A B}$ rotations in the Galileon space, and $K_{\mu A}$ generate rotations between the Minkowski and the Galileon space. This picture is strongly reminiscent of the probe brane construction of DBI and Galileon actions [30]. It should, however, be treated with some care: it is, for instance, not a priori guaranteed that the "metric" $g_{A B}$ is nonsingular or that all the generators $Q_{A B}$ are linearly independent, or even nonzero.

This is our main result, which is fully general, only assuming that the physical symmetry generators $Q_{i}$ commute with the whole Poincaré group and that all the redundant generators are Lorentz vectors. Although we only discussed the Lie algebra of symmetry generators so far, for spontaneously broken symmetries this fixes the action and thus all physical observables, up to a set of lowenergy effective couplings [12]. It is necessary to clarify, though, for which of the found Lie algebras a nontrivial action in fact exists.

Generalized Dirac-Born-Infeld theory.-To find an answer to the above question, let us first assume that the metric $g_{A B}$ is nonsingular. Its inverse $g^{A B}$ must also be invariant under the representation $T_{i}$. The generators $Q_{i}$ can then be redefined as

$$
\tilde{Q}_{i} \equiv Q_{i}+d_{A i} g^{A B} Q_{B} ;
$$

their commutators with other generators [cf. Eq. (6)] reduce to

$$
\begin{aligned}
{\left[\tilde{Q}_{i}, K_{\mu A}\right] } & =\left(t_{i}\right)_{A}^{B} K_{\mu B}, \quad\left[\tilde{Q}_{i}, Q_{A}\right]=\left(t_{i}\right)_{A}^{B} Q_{B}, \\
{\left[\tilde{Q}_{i}, Q_{A B}\right] } & =\left(t_{i}\right)_{A}^{C} Q_{C B}+\left(t_{i}\right)_{B}^{C} Q_{A C},
\end{aligned}
$$

where $\left(t_{i}\right)_{B}^{A} \equiv-i e_{B i}^{A}$ is a linear representation of $Q_{i}$. Since $\tilde{Q}_{A} \equiv a_{A}^{i} \tilde{Q}_{i}=0$, the scalar generators split into two sets: the shift generators $Q_{A}$ and those of $\tilde{Q}_{i}$ that are nonzero. $\left(Q_{A B}\right.$ are included among these thanks to $\tilde{Q}_{A B} \equiv$ $c_{A B}^{i} \tilde{Q}_{i}=Q_{A B}$.) The $\tilde{Q}_{i}$ still define a closed Lie algebra, $\left[\tilde{Q}_{i}, \tilde{Q}_{j}\right]=i f_{i j}^{k} \tilde{Q}_{k}$. Owing to Eq. (8), the symmetry algebra has the structure of a semidirect sum, where the subalgebra of $\tilde{Q}_{i}$ acts on the other generators through the representation $t_{i}$. In this case, the symmetry structure of the theory is thus fixed by giving (i) the Lie algebra of $\tilde{Q}_{i}$, (ii) its representation $t_{i}$, and (iii) the metric $g_{A B}$.

To proceed towards a construction of concrete actions, we use the method of nonlinear realizations, known as the coset construction [31], in the version valid for spacetime symmetries [32]. Denoting the broken generators among the $\tilde{Q}_{i}$ as $\tilde{Q}_{a}$, we parametrize the coset space as

$$
U(x, \theta, \xi) \equiv e^{i x^{\mu} P_{\mu}} e^{i \theta^{A} Q_{A}} e^{i \xi^{\mu A} K_{\mu A}} e^{i \theta^{a} \tilde{Q}_{a}} .
$$

Invariant actions can be built using the Maurer-Cartan (MC) form $\omega \equiv-i U^{-1} d U$. This can be decomposed in the basis of symmetry generators, the most interesting components for our purposes being $\omega_{P}^{\mu} P_{\mu}$ and $\omega_{Q}^{A} Q_{A}$. Introducing the shorthand notation $\hat{c h}(x) \equiv \cosh \sqrt{x}$ and $\hat{s h}(x) \equiv \sinh \sqrt{x} / \sqrt{x}$, and the matrices $\Pi_{\mu}^{\nu} \equiv g_{A B} \xi_{\mu}^{A} \xi^{\nu B}$ and $\amalg_{A}^{B} \equiv g_{A C} \xi^{\mu C} \xi_{\mu}^{B}$, these components of the MC form can be expressed as

$$
\begin{aligned}
\omega_{P}^{\mu} & =d x^{\nu}(\hat{c h} \Pi)_{\nu}^{\mu}-d \theta^{A} g_{A B} \xi^{\nu B}(\hat{s h} \Pi)_{\nu}^{\mu}, \\
\omega_{Q}^{A} & =\left(e^{-i \theta^{a} t_{a}}\right)_{B}^{A}\left[d \theta^{C}(\hat{c h} \amalg)_{C}^{B}-d x^{\mu} \xi_{\mu}^{C}(\hat{s h} \amalg)_{C}^{B}\right] .
\end{aligned}
$$

The latter can be used to eliminate the unphysical fields $\xi^{\mu A}$ by imposing an inverse Higgs constraint (IHC) [26,33]. Setting $\omega_{Q}^{A}=0$, the $\xi^{\mu A}$ are thus given implicitly by solving the algebraic equation $\partial_{\mu} \theta^{A}=\xi_{\mu}^{B}(\hat{s h} \amalg / \hat{c h} \amalg)_{B}^{A}$. The $\omega_{P}^{\mu}$ defines a covariant vielbein $e_{\mu}^{\alpha}$ by $\omega_{P}^{\alpha} \equiv e_{\mu}^{\alpha} d x^{\mu}$. This in turn leads to the metric $G_{\mu \nu} \equiv g_{\alpha \beta} e_{\mu}^{\alpha} e_{\nu}^{\beta}$. The leading-order action is obtained by integrating the invariant volume element $d^{4} x \sqrt{-|G|}$ and upon solving the IHC becomes

$$
S_{\mathrm{DBI}}=\int d^{4} x \sqrt{-\left|g_{\mu \nu}-g_{A B} \partial_{\mu} \theta^{A} \partial_{\nu} \theta^{B}\right|} .
$$

This is the action of the multiflavor DBI theory as outlined, e.g., in Ref. [16]. It describes a four-dimensional brane embedded in a $(4+N)$-dimensional flat spacetime, with the induced metric $G_{\mu \nu}$ on the brane. The $N$ NG modes $\theta^{A}$ arise from the $N$ spontaneously broken translations $Q_{A}$ in the extra dimensions. In the case of $N=1$, Eq. (11) can be cast as $S_{\mathrm{DBI}}=\int d^{4} x \sqrt{1-v\left(\partial_{\mu} \theta\right)^{2}}$, where $v \equiv g_{A=1, B=1}[13]$.

The multiflavor DBI theory discussed in Ref. [16] is based on the assumption that the extra-dimensional rotations generated by $Q_{A B}$ remain unbroken and that there are no other Lorentz-scalar symmetry generators apart from $Q_{A}$ and $Q_{A B}$. These assumptions are not required in our construction. The presence of other broken generators than $Q_{A}$ leads to additional NG modes $\theta^{a}$. While these do not enter the action (11), they do appear in the general DBI-like action, taking the form

$$
S_{\text {DBI-like }}=\int d^{4} x \sqrt{-|G|} \mathcal{L}_{\text {inv }}\left(\theta^{A}, \theta^{a}\right),
$$

where $\mathcal{L}_{\text {inv }}$ is an invariant Lagrangian density, built using the remaining components of the MC form: $\omega_{J}^{\mu \nu}, \omega_{K \mu}^{A}, \omega_{\tilde{Q}}^{i}$. One can, e.g., take as $\mathcal{L}_{\text {inv }}$ a Lagrangian for $\theta^{a}$ alone, constructed using standard methods [31], provided that indices inside are contracted using $G_{\mu \nu}$. The simplest example of such a theory corresponds to taking $N=1$ and a single, spontaneously broken generator $\tilde{Q}$ with the 
associated NG field $\phi$. The minimal interaction between $\theta$ and $\phi$ arises from the action

$$
S_{\text {DBI-like }}=\int d^{4} x \sqrt{-|G|} G^{\mu \nu} \partial_{\mu} \phi \partial_{\nu} \phi,
$$

where $G_{\mu \nu}=g_{\mu \nu}-v \partial_{\mu} \theta \partial_{\nu} \theta$.

Generalized Galileon theory.-To concretely work out another infinite class of theories that fit into our general framework, let us assume that $d_{A i}=0$. This assumption is natural: by means of the Jacobi identities, it implies $g_{A B}=0$ (see the Supplemental Material [29]) and hence makes $Q_{i}$ and $K_{\mu A}$ form a closed Lie algebra. This is the most general Lie algebra structure where both $Q_{i}$ and $K_{\mu A}$ generate an internal symmetry.

For the sake of simplicity, we will for the moment also assume that $c_{A B}^{i}=0$, that is, $Q_{A B}=0$. Such systems are then described by an extremely simple structure,

$$
\begin{gathered}
{\left[P_{\mu}, K_{\nu A}\right]=i g_{\mu \nu} Q_{A},} \\
{\left[Q_{i}, K_{\mu A}\right]=\left(t_{i}\right)_{A}^{B} K_{\mu B}, \quad\left[Q_{i}, Q_{A}\right]=\left(t_{i}\right)_{A}^{B} Q_{B},}
\end{gathered}
$$

along with $\left[Q_{A}, Q_{B}\right]=\left[K_{\mu A}, K_{\nu B}\right]=\left[K_{\mu A}, Q_{B}\right]=0$. All the commutators are now determined by specifying (i) the algebra of generators $Q_{i}$ and (ii) its Abelian ideal generated by $Q_{A}$. Finally, we will assume that, as for the DBI-like theories, all the generators $Q_{i}$ can be split into subsets $\tilde{Q}_{i}$ and $Q_{A}$, such that the $\tilde{Q}_{i}$ s themselves form a closed subalgebra [34].

The action for such theories can be obtained as above, using the parametrization (9) of the coset space. In this parametrization, we obtain the Galileon-like transformation rule under $Q_{A}$ and $K_{\mu A}, \theta^{A} \rightarrow \theta^{A}+\alpha^{A}+\beta_{\mu}^{A} x^{\mu}$. The generators $\tilde{Q}_{i}$, though, act on $\theta^{A}$ and $\xi^{\mu A}$ linearly through the matrices $t_{i}$. The only nontrivial pieces of the MC form are

$$
\begin{aligned}
\omega_{K \mu}^{A} & =\left(e^{-i \theta^{a} t_{a}}\right)_{B}^{A} d \xi_{\mu}^{B}, \\
\omega_{Q}^{A} & =\left(e^{-i \theta^{a} t_{a}}\right)_{B}^{A}\left(d \theta^{B}-\xi_{\mu}^{B} d x^{\mu}\right),
\end{aligned}
$$

together with the $\mathrm{MC}$ form for $\tilde{Q}_{i}, \omega_{\tilde{Q}}^{i}$.

The auxiliary fields $\xi^{\mu A}$ are eventually eliminated by using the IHC $\omega_{Q}^{A}=0$, that is, $\xi_{\mu}^{A}=\partial_{\mu} \theta^{A}$. It turns out that the whole class of theories possesses a set of WZ terms, invariant under the symmetry only up to a surface term [28]

$$
\mathcal{L}_{k}^{\mathrm{WZ}}=c_{A_{1} \ldots A_{k}} \theta^{A_{1}} G_{k-1}^{A_{2} \ldots A_{k}},
$$

where $G_{0} \equiv 1$ and $G_{k}$ for $k=1,2,3,4$ are defined by

$$
\begin{aligned}
G_{k}^{A_{1} \ldots A_{k}} \equiv & \frac{1}{(4-k) !} \epsilon_{\alpha_{1} \ldots \alpha_{k} \mu_{k+1} \ldots \mu_{4}} \epsilon^{\beta_{1} \ldots \beta_{k} \mu_{k+1} \ldots \mu_{4}} \\
& \times\left(\partial_{\beta_{1}} \partial^{\alpha_{1}} \theta^{A_{1}}\right) \ldots\left(\partial_{\beta_{k}} \partial^{\alpha_{k}} \theta^{A_{k}}\right)
\end{aligned}
$$

$c_{A_{1} \ldots A_{k}}$ must be fully symmetric invariant tensors of the representation $t_{i}$. Existing multi-Galileon Lagrangians [7] correspond to the special case where the generators $\tilde{Q}_{i}$ are unbroken, forming a compact Lie algebra such as $\mathrm{SO}(N)$ or $\mathrm{SU}(N)$. For an $\mathrm{SO}(N)$ vector of fields $\theta^{A}$, for instance, only $\mathcal{L}_{2}^{\mathrm{WZ}}$ and $\mathcal{L}_{4}^{\mathrm{WZ}}$ exist, for which, respectively, $c_{A B}=\delta_{A B}$ and $c_{A B C D}=\delta_{A B} \delta_{C D}+\delta_{A C} \delta_{B D}+\delta_{A D} \delta_{B C}$. Equation (16) is fully general in that it applies to an arbitrary Lie algebra, generated by $\tilde{Q}_{i}$, and its arbitrary real (finite-dimensional) representation $t_{i}$ and allows for $\tilde{Q}_{i}$ to be spontaneously broken.

As for the DBI theory (11), the WZ terms (16) are blind to the $\theta^{a}$ fields, regardless of the symmetry-breaking pattern. Interactions between the Galileon and nonGalileon sector enter only through the strictly invariant part of the Lagrangian, and can be constructed by taking a product of $\omega_{K \mu}^{A}$ and $\omega_{\tilde{Q}}^{a}$ or their derivatives. These invariant Lagrangians realize the enhanced soft limit of scattering amplitudes of the Galileon modes trivially in that they only contain operators with at least two derivatives on each $\theta^{A}$.

Twisted Galileon theory.-We shall now restore the generators $Q_{A B}$, otherwise keeping the same assumptions as in the construction of the generalized Galileon theory. The resulting Lie algebra features a twisted commutator,

$$
\left[K_{\mu A}, K_{\nu B}\right]=i g_{\mu \nu} Q_{A B} .
$$

Equation (14) still holds, and $Q_{A B}$ transforms as a rank-2 tensor under the adjoint action of $Q_{i}$. All the generators $Q_{A}$ and $Q_{A B}$ commute with each other and with $K_{\mu C}$.

The coset construction proceeds as above upon the replacement $e^{i \theta^{A} Q_{A}} \rightarrow e^{i \theta^{A} Q_{A}} e^{(i / 2) \theta^{A B} Q_{A B}}$ in Eq. (9), where $\theta^{A B}$ are NG fields for those of the $Q_{A B}$ generators that are spontaneously broken. All our conclusions reached in the discussion of the generalized Galileon theory-Eq. (15) and below-remain valid. However, the new component of the MC form

$$
\begin{aligned}
\omega_{Q}^{A B}= & \left(e^{-i \theta^{a} t_{a}}\right)_{C}^{A}\left(e^{-i \theta^{b} t_{b}}\right)_{D}^{B} \\
& \times\left[d \theta^{C D}+\frac{1}{2}\left(\xi_{\mu}^{C} d \xi^{\mu D}-\xi_{\mu}^{D} d \xi^{\mu C}\right)\right]
\end{aligned}
$$

automatically induces interactions of the Galileon modes $\theta^{A}$ with the non-Galileon modes $\theta^{A B}$. The entanglement of the $\theta^{A}$ and $\theta^{A B}$ sectors is due to the transformation of $\theta^{A B}$ under $K_{\mu A}$, which reads $\theta^{A B} \rightarrow \theta^{A B}-\frac{1}{2}\left(\beta_{\mu}^{A} \xi^{\mu B}-\beta_{\mu}^{B} \xi^{\mu A}\right)$. Upon imposing the IHC, $\xi_{\mu}^{A}=\partial_{\mu} \theta^{A}$, a Lagrangian built up from $\omega_{Q}^{A B}$ will contain less than two derivatives per $\theta^{A}$.

The simplest example of such a theory is obtained by taking $\mathrm{SO}(2)$ for the algebra of $\tilde{Q}_{i}$ and assuming that it remains unbroken. Then, $Q_{A}$ reduces to an $\mathrm{SO}(2)$ vector $Q_{1}, Q_{2}$, and $Q_{A B}$ to an $\mathrm{SO}(2)$ singlet $Q_{12}$. The resulting interaction Lagrangian, complementing the pure Galileon WZ terms, reads 
$\mathcal{L}_{\text {twist }}=\frac{1}{2}\left[\partial_{\mu} \theta^{12}+\frac{1}{2}\left(\partial^{\nu} \theta^{1} \partial_{\mu} \partial_{\nu} \theta^{2}-\partial^{\nu} \theta^{2} \partial_{\mu} \partial_{\nu} \theta^{1}\right)\right]^{2}$.

Interestingly, the scattering amplitudes of $\theta^{1}, \theta^{2}$ in this theory do not have an enhanced soft limit, in spite of the Galilean shift symmetry, acting on these fields. This can be attributed to the cubic part of the Lagrangian (20), which leads to collinear singularities in the soft limit [13]. We expect the same behavior for all twisted Galileon theories.

Conclusions. - Under only very mild assumptions, we have found the most general symmetry structure, admitting a set of generators that, though spontaneously broken, do not give rise to $\mathrm{NG}$ bosons. We thereby mapped the landscape of possible theories of multiple massless scalars whose scattering amplitudes exhibit an enhanced soft limit [13]. Our results reduce the construction of such theories to the problem of finding an affine representation of the Lie algebra of physical internal symmetries and an invariant metric on its target space.

Intriguingly, our analysis singles out the multi-Galileon and multiflavor DBI theories as possibly the only systems where the scattering amplitudes of all the modes have an enhanced soft limit. We stress, however, that our discussion of the solutions to the Lie-algebraic constraints was not exhaustive. The gap for some options not considered here is narrowed down, or closed, in the Supplemental Material [29]. This includes systems where the metric $g_{A B}$ is singular but nonzero, algebras of the type (14) where the nonGalileon generators $\tilde{Q}_{i}$ do not form a closed subalgebra, and algebras of the type (18) where the generators $Q_{A B}$ are not linearly independent of $Q_{A}$.

Finally, our analysis was restricted to Lorentz-invariant theories, yet our Lie-algebraic approach can be applied equally well to nonrelativistic systems. In condensedmatter physics, there is a plethora of naturally occurring redundant symmetries such as Galilei boosts or rotations in crystalline solids. The need to understand the consequences of such symmetries provides a strong motivation for an extension of the present results, which will be addressed in our future work.

We thank Torsten Schoeneberg and Qiaochu Yuan for advice regarding the structure of Lie algebras possessing an Abelian ideal. This work has been supported by a ToppForsk-UiS Grant No. PR-10614.

*mark.bogers@uis.no

†tomas.brauner@uis.no

[1] T. L. Curtright and D. B. Fairlie, arXiv:1212.6972; C. Deffayet and D. A. Steer, Classical Quantum Gravity 30, 214006 (2013).

[2] G. W. Horndeski, Int. J. Theor. Phys. 10, 363 (1974); D. B. Fairlie, J. Govaerts, and A. Morozov, Nucl. Phys. B373, 214 (1992).
[3] A. Nicolis, R. Rattazzi, and E. Trincherini, Phys. Rev. D 79, 064036 (2009).

[4] L. Lombriser and A. Taylor, J. Cosmol. Astropart. Phys. 03 (2016) 031; L. Lombriser and N. A. Lima, Phys. Lett. B 765, 382 (2017).

[5] T. Baker, E. Bellini, P. G. Ferreira, M. Lagos, J. Noller, and I. Sawicki, Phys. Rev. Lett. 119, 251301 (2017); P. Creminelli and F. Vernizzi, Phys. Rev. Lett. 119, 251302 (2017); J. Sakstein and B. Jain, Phys. Rev. Lett. 119, 251303 (2017); J. M. Ezquiaga and M. Zumalacárregui, Phys. Rev. Lett. 119, 251304 (2017).

[6] E. Witten, Nucl. Phys. B223, 422 (1983); E. D'Hoker and S. Weinberg, Phys. Rev. D 50, R6050 (1994); E. D'Hoker, Nucl. Phys. B451, 725 (1995).

[7] G. Goon, K. Hinterbichler, A. Joyce, and M. Trodden, J. High Energy Phys. 06 (2012) 004.

[8] G. Goon, K. Hinterbichler, A. Joyce, and M. Trodden, J. High Energy Phys. 11 (2016) 100.

[9] K. Hinterbichler, M. Trodden, and D. Wesley, Phys. Rev. D 82, 124018 (2010).

[10] C. de Rham, M. Fasiello, and A. J. Tolley, Phys. Lett. B 733, 46 (2014); C. de Rham, L. Keltner, and A. J. Tolley, Phys. Rev. D 90, 024050 (2014); K. Kampf and J. Novotný, J. High Energy Phys. 10 (2014) 006.

[11] K. Hinterbichler and A. Joyce, Phys. Rev. D 92, 023503 (2015); J. Noller, V. Sivanesan, and M. von Strauss, Phys. Rev. D 92, 064009 (2015); J. Novotný, Phys. Rev. D 95, 065019 (2017).

[12] S. Weinberg, The Quantum Theory of Fields (Cambridge University Press, Cambridge, England, 1996), Vol. II.

[13] C. Cheung, K. Kampf, J. Novotný, and J. Trnka, Phys. Rev. Lett. 114, 221602 (2015).

[14] P. Benincasa, Int. J. Mod. Phys. A 29, 1430005 (2014); H. Elvang and Y.-t. Huang, arXiv:1308.1697; C. Cheung, arXiv:1708.03872.

[15] C. Cheung, K. Kampf, J. Novotný, C.-H. Shen, and J. Trnka, Phys. Rev. Lett. 116, 041601 (2016); A. Padilla, D. Stefanyszyn, and T. Wilson, J. High Energy Phys. 04 (2017) 015.

[16] C. Cheung, K. Kampf, J. Novotný, C.-H. Shen, and J. Trnka, J. High Energy Phys. 02 (2017) 020.

[17] C. Deffayet, S. Deser, and G. Esposito-Farèse, Phys. Rev. D 80, 064015 (2009); C. Deffayet, X. Gao, D. A. Steer, and G. Zahariade, Phys. Rev. D 84, 064039 (2011).

[18] G. L. Goon, K. Hinterbichler, and M. Trodden, J. Cosmol. Astropart. Phys. 07 (2011) 017; Phys. Rev. Lett. 106, 231102 (2011).

[19] A. Padilla, P. M. Saffin, and S.-Y. Zhou, J. High Energy Phys. 12 (2010) 031.

[20] A. Padilla and V. Sivanesan, J. High Energy Phys. 04 (2013) 032; V. Sivanesan, Phys. Rev. D 90, 104006 (2014); E. Allys, Phys. Rev. D 95, 064051 (2017).

[21] A. Padilla, P. M. Saffin, and S.-Y. Zhou, Phys. Rev. D 83, 045009 (2011).

[22] S. R. Coleman and J. Mandula, Phys. Rev. 159, 1251 (1967).

[23] I. Low and A. V. Manohar, Phys. Rev. Lett. 88, 101602 (2002); H. Watanabe and H. Murayama, Phys. Rev. Lett. 110, 181601 (2013); K. Hinterbichler and A. Joyce, Int. J. Mod. Phys. D 23, 1443001 (2014); T. Griffin, K. T. Grosvenor, 
P. Hořava, and Z. Yan, Commun. Math. Phys. 340, 985 (2015); Phys. Rev. Lett. 115, 241601 (2015).

[24] While there seems to be no general proof that enhancement of the soft limit requires redundant symmetry, all currently known examples of theories with an enhanced soft limit possess one.

[25] H. Watanabe and A. Vishwanath, Proc. Natl. Acad. Sci. U.S.A. 111, 16314 (2014); I. Z. Rothstein and P. Shrivastava, J. High Energy Phys. 05 (2018) 014.

[26] E. Ivanov and V. I. Ogievetsky, Teor. Mat. Fiz. 25, 164 (1975).

[27] In principle, the redundant generators may form a hierarchy of Lorentz vectors and higher-rank tensors, leading to multiply enhanced scattering amplitudes in the soft limit. This possibility is considered elsewhere [28].

[28] M. P. Bogers and T. Brauner, J. High Energy Phys. 05 (2018) 076.

[29] See Supplemental Material at http://link.aps.org/ supplemental/10.1103/PhysRevLett.121.171602 for a list of constraints from Jacobi identities and for a discussion of allowed Lie-algebraic structures that do not lead to physically interesting theories.

[30] C. de Rham and A. J. Tolley, J. Cosmol. Astropart. Phys. 05 (2010) 015.

[31] S. R. Coleman, J. Wess, and B. Zumino, Phys. Rev. 177, 2239 (1969); C. G. Callan, S. Coleman, J. Wess, and B. Zumino, Phys. Rev. 177, 2247 (1969).

[32] D. V. Volkov, Fiz. Elem. Chastits At. Yadra 4, 3 (1973); V. Ogievetsky, Acta Univ. Wratislav. 207, 117 (1974); in Proceedings of 10th Winter School of Theoretical Physics in Karpacz, 1973 (State Scientific Publishers, Warszawa, 1973).

[33] T. Brauner and H. Watanabe, Phys. Rev. D 89, 085004 (2014); R. Klein, D. Roest, and D. Stefanyszyn, J. High Energy Phys. 10 (2017) 051.

[34] The simplest example of a Lie algebra that does not satisfy this assumption is the Heisenberg algebra. Other examples are provided generally by Lie algebras possessing central charges. 